\title{
Collaboration Control of Fractional-Order Multiagent Systems with Sampling Delay
}

\author{
Hong-yong Yang, ${ }^{1,2}$ Lei Guo, ${ }^{2}$ Banghai $X u,{ }^{1}$ and Jian-zhong Gu${ }^{1}$ \\ ${ }^{1}$ School of Information and Electrical Engineering, Ludong University, Yantai 264025, China \\ ${ }^{2}$ National Key Laboratory on Aircraft Control Technology, Beihang University, Beijing 100191, China \\ Correspondence should be addressed to Hong-yong Yang; hyyang_ld@yahoo.com.cn
}

Received 2 February 2013; Accepted 16 April 2013

Academic Editor: Yang Yi

Copyright (c) 2013 Hong-yong Yang et al. This is an open access article distributed under the Creative Commons Attribution License, which permits unrestricted use, distribution, and reproduction in any medium, provided the original work is properly cited.

\begin{abstract}
Because of the complexity of the practical environments, many distributed multiagent systems cannot be illustrated with the integerorder dynamics and can only be described with the fractional-order dynamics. In this paper, collaboration control problems of continuous-time networked fractional-order multiagent systems via sampled control and sampling delay are investigated. Firstly, the sampled-data control of multiagent systems with fractional-order derivative operator is analyzed in a directed weighted network ignoring sampling delay. Then, the collaborative control of fractional-order multiagent systems with sampled data and sampling delay is studied in a directed and symmetrical network. Many sufficient conditions for reaching consensus with sampled data and sampling delay are obtained. Some numerical simulations are presented to illustrate the utility of our theoretical results.
\end{abstract}

\section{Introduction}

In recent years, consensus problems in distributed networked multiagent systems have attracted increasing attention of more and more researches including control theory, mathematics, biology, physics, computer science, and robotics. The applications of multiagent systems are extensive, ranging from multiple space-craft alignment, heading direction in flocking behavior, distributed computation, and rendezvous of multiple vehicles. Based on certain quantities of interest, collaboration control problems of agent systems have been studied by many researchers and many important results have been achieved in much literature [1-8].

With the development of digital sensors and controllers, in many cases that the system itself is a continuous process, the synthesis of control law can only use the data sampled at the discrete sampling instants. Compare to continuoustime systems with continuous-time controller, continuoustime systems via sampled control have many advantages, such as flexibility, robustness, and low cost. Therefore, sampled control for continuous-time system is more coincident with applications in our real life. Robots, vehicles, airplanes, satellites, and almost all of the modern artificial products are controlled by digital controller where continuous signals are transferred into discrete ones. For consensus problems of continuous-time multiagent systems via sampled control, some interesting results about consensus problem for multiagent system have been reported [9-15]. However, in the many real applications, we always want to find how large the sampling period should be chosen to guarantee that the system runs well. This requires us to look for an upper bound of sampling period. Moreover, sampling delay of the system cannot be ignored and sometimes may play a key role in the stability analysis of the networks. Therefore, we will also study the case when sampling delay exists.

The important results of the above literature focus the consensus problems of multiagent systems with integer-order dynamical equation. In the complex environment, many phenomena cannot be explained by the framework of integerorder dynamics, for example, the synchronized motion of agents in complex environments such as macromolecule fluids and porous media [16-18]. Under these circumstances, many dynamic characteristics of natural phenomena can only be described in the dynamics of fractional-order (noninteger order) behavior, for example: flocking movement and food searching by means of the individual secretions 
and microbial, submarine underwater robots in the bottom of the sea with a large number of microorganisms and viscous substances, unmanned aerial vehicles running in the complex space environment $[19,20]$. Cao et al. $[21,22]$ studied distribution coordination of multiagent systems with fractional-order dynamics firstly and gave the relationship between the number of individuals and the fractional order in the stable multiagent systems. However, to the best of our knowledge, there are few researches done on the coordination control of fractional-order multiagent systems via sampled data.

In this paper, we investigate the consensus of fractionalorder multiagent systems (FOMAS) with sampled-data control. Because few methods are presented to analyze the fractional-order systems with sampling delay, the problems of the fractional-order systems with sampling delay and sampled data will become more difficult. The main innovation of this paper lies in the study on the distributed coordination of FOMAS with sampled data and sampling delay. The rest of the paper is organized as follows. In Section 2, we recall some basic definitions about fractional calculus. In Section 3, some preliminaries about graph theory, fractional-order coordination model of multiagent systems are shown out. A distributed coordination algorithm for FOMAS with sampled data control is studied in Section 4. Section 5 presents the consensus of FOMAS with sampled data and sampling delay. In Section 6, numerical examples are simulated to verify the theoretical analysis. Conclusions are finally drawn in Section 7.

\section{Fractional Calculus}

Fractional derivatives provide an excellent instrument to describe the memory and hereditary features of various materials and processes. Fractional calculus also appears in the control of dynamical systems, when the controlled system and the controller are described by a fractionalorder differential equation. This is the main advantage of fractional derivatives in comparison with classical integerorder models, in which such effects are in fact neglected. The advantages of fractional-order derivatives become evident in modeling mechanical and electrical characteristics of real materials, as well as in many fields to describe the rheological properties of rocks.

Fractional operator plays an important role in modern science, which is used as a generalization of integration and differentiation with noninteger order fundamental operator ${ }_{a} D_{t}^{p}$, where a and $\mathrm{t}$ are the limits of the operation and $p \in R$. The continuous integrodifferential operator is defined as

$$
{ }_{a} D_{t}^{p}= \begin{cases}\frac{d^{p}}{d t^{p}}, & p>0, \\ 1, & p=0 \\ \int_{a}^{t}(d \theta)^{-p}, & p<0\end{cases}
$$

Lemma 1 (see $[5]) .0$ is a simple eigenvalue of Laplacian
matrix $L$, and $X_{0}=C[1,1, \ldots, 1]^{T}$ is corresponding right
eigenvector, that is, $L X_{0}=0$, if and only if the digraph $\mathscr{G}=$
$(V, E, A)$ has a globally reachable node.
Lemma 1 (see $[5]) .0$ is a simple eigenvalue of Laplacian
matrix $L$, and $X_{0}=C[1,1, \ldots, 1]^{T}$ is corresponding right
eigenvector, that is, $L X_{0}=0$, if and only if the digraph $\mathscr{G}=$
$(V, E, A)$ has a globally reachable node.

Lemma 1 (see $[5]) .0$ is a simple eigenvalue of Laplacian
matrix $L$, and $X_{0}=C[1,1, \ldots, 1]^{T}$ is corresponding right
eigenvector, that is, $L X_{0}=0$, if and only if the digraph $\mathscr{G}=$
$(V, E, A)$ has a globally reachable node.

Lemma 1 (see $[5]) .0$ is a simple eigenvalue of Laplacian
matrix $L$, and $X_{0}=C[1,1, \ldots, 1]^{T}$ is corresponding right
eigenvector, that is, $L X_{0}=0$, if and only if the digraph $\mathscr{G}=$
$(V, E, A)$ has a globally reachable node.

Three definitions most frequently used for the general fractional operators are the Grünwald-Letnikov (GL) definition, the Riemann-Liouville (RL), and the Caputo definition [16-18]. The GL definition is given by

$$
{ }_{a}^{G} D_{t}^{p} f(t)=\lim _{h \rightarrow 0} h^{-p} \sum_{k=0}^{[(t-a) / h]}(-1)^{k}\left(\begin{array}{l}
p \\
k
\end{array}\right) f(t-k h)
$$

where $[\cdot]$ means the integer part, and $\left(\begin{array}{l}p \\ k\end{array}\right)$ is fractional binomial coefficients. The RL definition is given as

$$
{ }_{a}^{R} D_{t}^{p} f(t)=\frac{1}{\Gamma(n-p)} \frac{d^{n}}{d t^{n}} \int_{a}^{t} \frac{f(\theta)}{(t-\theta)^{1+p-n}} d \theta
$$

for $(n-1<p<n)$ and where $\Gamma(\cdot)$ is the Gamma function. The Caputo definition can be written as

$$
{ }_{a}^{C} D_{t}^{p} f(t)=\frac{1}{\Gamma(p-n)} \int_{a}^{t} \frac{f^{(n)}(\theta)}{(t-\theta)^{1+p-n}} d \theta
$$

The initial conditions for the fractional order differential equations with the Caputo derivatives are in the same form as a simple notation $f^{(p)}$ is used to replace ${ }_{a} D_{t}^{p} f(t)$.

\section{Problem Statement}

Assume that multiagent system consists of $n$ autonomous agents then connected relations between the agents constitute a network topology $\mathscr{G}$. Assume $\mathscr{G}=\{V, E, A\}$ represents a directed weighted graph, in which $V=\left\{v_{1}, v_{2}, \ldots, v_{n}\right\}$ represents a collection of $n$ nodes, and its set of edges is $E \subseteq V \times V$. The node indexes belong to a finite index set $I=\{1,2, \ldots, n\}$, with adjacency matrix $A=\left[a_{i l}\right] \in R^{n \times n}$ with weighted adjacency elements $a_{i l} \geq 0$. An edge of the weighted diagraph $\mathscr{G}$ is denoted by $e_{i l}=\left(v_{i}, v_{l}\right) \in E$. We assume that the adjacency element $a_{i l}>0$ when $e_{i l} \in E$; otherwise, $a_{i l}=0$. The set of neighbors of a node $i$ is denoted by $N_{i}=\left\{l \in I: a_{i l}>0\right\}$.

Let $\mathscr{G}$ be a weighted digraph without self-loops, that is, let $a_{i i}=0$, and matrix $D=\operatorname{diag}\left\{d_{1}, d_{2}, \ldots, d_{n}\right\}$ be the diagonal matrix with the diagonal elements $d_{i}=\sum_{l=1}^{n} a_{i l}$ representing the sum of the elements in the ith row of matrix $A$. The Laplacian matrix of the weighted digraph $\mathscr{G}$ is defined as $L=$ $D-A$. For two nodes $i$ and $l$, there is subscript set $\left\{l_{1}, l_{2}, \ldots l_{k}\right\}$ satisfying $a_{i l_{1}}>0, a_{l_{1} l_{2}}>0, \ldots, a_{l_{k} l}>0$, and then there is a directed linked path between node $i$ and node $l$ which is used for the information transmission, also we can say that node $i$ can receive the information from node $l$. If node $i$ can find a path to reach any node of the graph, then node $i$ is globally reachable from every other node in the digraph. In this paper, the directed graph and directed symmetrical graph for fractional-order multiagent systems will be considered. that for the integer-order differential equations. In this paper, 
Given that the dynamics of multiagent systems indicated with fractional derivative in the complex environments, the fractional dynamical equations are defined as

$$
x_{i}^{(\alpha)}=u_{i}(t), \quad i=1, \ldots, n,
$$

where $x_{i}(t) \in R$ and $u_{i}(t) \in R$ represent the $i$ th agent's state and control input, respectively, and $x_{i}^{(\alpha)}$ represents the $\alpha(\alpha>$ 0 ) order fractional derivative. Assume that the following control protocols are used in multiagent systems:

$$
u_{i}(t)=-\gamma \sum_{l \in N_{i}} a_{i l}\left[x_{i}(t)-x_{l}(t)\right], \quad i=1, \ldots, n,
$$

where $a_{i l}$ represents the $(i, l)$ elements of adjacency matrix $A, \gamma>0$ is control gain, and $N_{i}$ represents the neighbors collection of the $i$ th agent.

Suppose that for any initial value of the system, the states of autonomous agents meet $\lim _{t \rightarrow \infty}\left(x_{i}(t)-x_{l}(t)\right)=0$, for $i, l \in I$, and then multiagent systems asymptotically reach consensus.

\section{Sampled Control of FOMAS}

Suppose that the sampling period is $h$; then the discrete-time dynamics of multiagent systems with fractional derivative can be rewritten as

$$
x_{i}(k+1)=\alpha x_{i}(k)+h^{\alpha} u_{i}(k), \quad i=1, \ldots, n,
$$

we have

$$
X(k+1)=\Psi X(k)
$$

where $X(k)=\left[x_{1}(k), x_{2}(k), \ldots, x_{n}(k)\right]^{T}, \Psi=\alpha I_{n}-h^{\alpha} \gamma L$, and $I_{n}$ is a unit matrix with $n$-dimensions. If the norm of matrix $\Psi$ is satisfying $\|\Psi\|<1$, the fractional-order discrete-time multiagent system (8) will asymptotically reach consensus.

Theorem 2. Suppose that multiagent systems are composed of $n$ independent agents, whose connection network topology is directed, and there is a global reachable node. Then fractionalorder multiagent system (7) with sampled data can asymptotically reach consensus, if $\alpha<1$ and

$$
\begin{gathered}
h<\min \left\{\left[\frac{\alpha \operatorname{Re}\left(\lambda_{i}\right)}{\gamma\left|\lambda_{i}^{2}\right|}+\frac{1}{\gamma\left|\lambda_{i}\right|} \sqrt{1-\alpha^{2}\left(\frac{\operatorname{Im}\left(\lambda_{i}\right)}{\left|\lambda_{i}\right|}\right)^{2}}\right]^{1 / \alpha},\right. \\
i=2, \ldots, n\},
\end{gathered}
$$

where $\lambda_{i}$ is the eigenvalue of the Laplacian matrix $L$.

Proof. Since the spectral radius $\rho$ of the matrix $\Psi$ is satisfying $\rho \leq\|\Psi\|$, we will require the spectral radius $\rho<1$. By the definition $\rho(\Psi)=\max \left\{\left|\eta_{i}\right|, i=1, \ldots, n\right\}$ where $\eta_{i}$ is the characteristic value of the matrix $\Psi$, we should calculate the characteristic value $\eta_{i}$ of the matrix $\Psi$ with $\left|\eta_{i}\right|<1$.

For any matrix $L$, there exists a unitary matrix $P$ satisfying

$$
\Lambda=P L P^{H},
$$

where conjugate matrix $P^{H}=P^{-1}, \Lambda$ is an upper triangular matrix, and its diagonal elements $\lambda_{1}, \ldots, \lambda_{n}$ are the eigenvalues of matrix $L$. From $P \Psi P^{H}=\alpha I_{n}-h^{\alpha} \gamma \Lambda$, we can obtain the characteristic values of matrix $\Psi$ being

$$
\eta_{i}=\alpha-h^{\alpha} \gamma \lambda_{i}, \quad i=1, \ldots, n .
$$

Suppose that the connection network topology of FOMAS is directed, and there is a global reachable node; then $\operatorname{Rank}(L)=$ $n-1$ and $\lambda=0$ is a single eigenvalue of Laplacian matrix $L$. Suppose $\lambda_{1}=0$, we have $\eta_{1}=\alpha-h^{\alpha} \gamma \lambda_{1}=\alpha$. From the stability requirement of the system (7), we obtain $\alpha<1$.

For other characteristic values of Laplacian matrix $L$ with $\lambda_{i} \neq 0(i \neq 1)$, the corresponding characteristic values of matrix $\Psi$ are being $\eta_{i}=\alpha-h^{\alpha} \gamma \lambda_{i}, i=2, \ldots, n$. Let $\lambda=$ $\operatorname{Re}\left(\lambda_{i}\right)+j \operatorname{Im}\left(\lambda_{i}\right)$ (where $j$ is complex number unit), and then the corresponding characteristic values, for $i=2, \ldots, n$, are

$$
\eta_{i}=\alpha-h^{\alpha} \gamma\left(\operatorname{Re}\left(\lambda_{i}\right)+j \operatorname{Im}\left(\lambda_{i}\right)\right)
$$

From $\left|\eta_{i}\right|<1$, we can obtain

$$
\alpha^{2}-2 \alpha h^{\alpha} \gamma \operatorname{Re}\left(\lambda_{i}\right)+h^{2 \alpha} \gamma^{2}\left|\lambda_{i}^{2}\right|<1 .
$$

It has

$$
h^{\alpha}<\frac{\alpha \operatorname{Re}\left(\lambda_{i}\right)}{\gamma\left|\lambda_{i}^{2}\right|}+\frac{1}{\gamma\left|\lambda_{i}\right|} \sqrt{1-\alpha^{2}\left(\frac{\operatorname{Im}\left(\lambda_{i}\right)}{\left|\lambda_{i}\right|}\right)^{2}}, \quad i=2, \ldots, n .
$$

Then, the condition of the fractional-order multiagent system (7) is obtained. The proof is finished.

Corollary 3. Suppose that multiagent systems are composed of $n$ independent agents, whose connection network topology is directed and symmetrical, and there is a global reachable node. Then FOMAS (7) can asymptotically reach consensus via sampled data, if $\alpha<1$ and

$$
h<\left(\frac{\alpha+1}{\gamma \lambda_{n}}\right)^{1 / \alpha}
$$

where $\lambda_{n}$ is the maximum eigenvalue of the Laplacian matrix $L$.

Corollary 4. Suppose multiagent systems are composed of $n$ independent agents, whose connection network topology is directed and symmetrical, and there is a global reachable node. Then multiagent system (7) with $\alpha=1$ can asymptotically reach consensus via sampled data, if

$$
h<\frac{2}{\gamma \lambda_{n}}
$$

where $\lambda_{n}$ is the maximum eigenvalue of the Laplacian matrix $L$. 
Remark 5. The system of Corollary 4 for fractional-order $\alpha=1$ becomes the first-order multiagent system. The consensus condition obtained in Corollary 4 is same as that in [9].

\section{Consensus of FOMAS with Sampled Data and Sampling Delay}

In the practical application, the sampled-data transferring will result in the communication delays. The sampling delays will affect the control features of the system and sometimes may play a key role in the stability analysis of the network. In this section, we will study the consensus of multiagent systems with sampled data and sampling delay.

Suppose that land the sampling period is $h$, the sampling delay is $\tau$. The sampled control protocols are used in multiagent systems as follows:

$u_{i}(t)$

$$
= \begin{cases}-\gamma \sum_{l \in N_{i}} a_{i l}\left[x_{i}(k-1)-x_{l}(k-1)\right], & t \in[k h, k h+\tau), \\ -\gamma \sum_{l \in N_{i}} a_{i l}\left[x_{i}(k)-x_{l}(k)\right], & t \in[k h+\tau, k h+h) .\end{cases}
$$

Based on (7) and (17), the dynamics of FOMAS with sampled data and sampling delay can be rewritten as

$$
\left(\begin{array}{c}
X(k+1) \\
X(k)
\end{array}\right)=\Phi\left(\begin{array}{c}
X(k) \\
X(k-1)
\end{array}\right),
$$

where

$$
\begin{gathered}
X(k)=\left[x_{1}(k), x_{2}(k), \ldots, x_{n}(k)\right]^{T}, \\
\Phi=\left(\begin{array}{cc}
\alpha I_{n}-\gamma\left(h^{\alpha}-h^{\alpha} \tau\right) L & -\gamma h^{\alpha} \tau L \\
I_{n} & 0
\end{array}\right),
\end{gathered}
$$

and $I_{n}$ is a unit matrix with $n$-dimensions. If the norm of matrix $\Phi$ is satisfying $\|\Phi\|<1$, the discrete-time FOMAS (18) will asymptotically reach consensus.

Lemma 6 (see [23, Hermite-Biehler Theorem]). Suppose the polynomial

$$
p(s)=p_{0}+p_{1} s+\cdots+p_{n} s^{n}
$$

Substituting $s=i \omega$ into the polynomial $p(s)$ yields

$$
p(\omega)=m(\omega)+i n(\omega)
$$

Then, the polynomial $p(s)$ is Hurwitz stability if and only if the related pair $m(\omega)$ and $n(\omega)$ is interlaced, and $m(0) n^{\prime}(0)-$ $m^{\prime}(0) n(0)>0$.

Theorem 7. Suppose that multiagent systems are composed of $n$ independent agents, whose connection network topology is directed and symmetrical, and there is a global reachable node.
Then FOMAS (18) with sampled data and sampling delay can asymptotically reach consensus, if $\alpha<1$ and

$$
\begin{gathered}
h<\left[\frac{1}{\gamma \tau \lambda_{n}}\right]^{1 / \alpha}, \quad \tau>\frac{1}{(3+\alpha)}, \\
h<\left[\frac{1+\alpha}{\gamma \lambda_{n}(1-2 \tau)}\right]^{1 / \alpha}, \quad \tau \leq \frac{1}{(3+\alpha)},
\end{gathered}
$$

where $\lambda_{n}$ is the maximum eigenvalue of the Laplacian matrix $L$.

Proof. In order to prove the asymptotical consensus of discrete-time systems (18), the spectral radius $\rho$ of the matrix $\Phi$ should be satisfied with $\rho(\Phi)<1$. Since $\rho(\Phi)=$ $\max \left\{\left|v_{i}\right|, i=1, \ldots, n\right\}$ where $\nu_{i}$ is the characteristic value of the matrix $\Phi$, we should calculate the characteristic value $v_{i}$ of the matrix $\Phi$ with $\left|\nu_{i}\right|<1$.

Suppose that the network topology of FOMAS is directed and symmetrical, and there is a global reachable node; then $\operatorname{Rank}(L)=n-1$ and $\lambda=0$ is a single eigenvalue of Laplacian matrix $L$. There exists an orthogonal matrix $P$ satisfying $\Lambda=$ $P L P^{T}$ where $P^{T}=P^{-1}, \Lambda$ is a diagonal matrix, and its diagonal elements $\lambda_{1}, \ldots, \lambda_{n}$ are the characteristic values of matrix $L$. Without loss of generality, we suppose $0=\lambda_{1}<\lambda_{2} \leq \cdots \leq$ $\lambda_{n}$. The characteristic equation of matrix $\Phi$ will be calculated as follows:

$$
\begin{aligned}
\operatorname{det} & \left(\nu I_{2 n \times 2 n}-\Phi\right) \\
= & \operatorname{det}\left(\begin{array}{cc}
\nu I_{n}-\alpha I_{n}+\gamma\left(h^{\alpha}-h^{\alpha} \tau\right) L & \gamma h^{\alpha} \tau L \\
-I_{n} & \nu I_{n}
\end{array}\right) \\
= & \operatorname{det}\left(\nu^{2} I_{n}-\nu\left(\alpha I_{n}-\gamma\left(h^{\alpha}-h^{\alpha} \tau\right) L\right)+\gamma h^{\alpha} \tau L\right) \\
= & \Pi_{i=1}^{n}\left[\nu^{2}-\nu \alpha+\left(\nu h^{\alpha}-v h^{\alpha} \tau+h^{\alpha} \tau\right) \gamma \lambda_{i}\right] .
\end{aligned}
$$

Let $a(\nu)=\nu^{2}-v \alpha+\left(\nu h^{\alpha}-v h^{\alpha} \tau+h^{\alpha} \tau\right) \gamma \lambda_{i}$. When $\lambda_{1}=0$ and $a(\nu)=\nu^{2}-\nu \alpha$, we can obtain the characteristic values $\nu_{1}=0$ and $\nu_{2}=\alpha$. From the stability requirement of the system (18), we obtain $\alpha<1$.

When $\lambda_{i}>0(i=2, \ldots, n)$, applying the bilinear transform $\nu=(s+1) /(s-1), a(\nu)$ can be converted into $b(s)$ as follows:

$$
\begin{aligned}
b(s)= & \left(1-\alpha+h^{\alpha} \gamma \lambda_{i}\right) s^{2}+2\left(1-h^{\alpha} \gamma \tau \lambda_{i}\right) s \\
& +\left(1+\alpha-h^{\alpha} \gamma \lambda_{i}+2 h^{\alpha} \tau \gamma \lambda_{i}\right) .
\end{aligned}
$$

Let $s=j \omega$, and the we have

$$
b(\omega)=m(\omega)+j n(\omega),
$$

where $m(\omega)=-\left(1-\alpha+h^{\alpha} \gamma \lambda_{i}\right) \omega^{2}+\left(1+\alpha-h^{\alpha} \gamma \lambda_{i}+2 h^{\alpha} \tau \gamma \lambda_{i}\right)$ and $n(\omega)=2\left(1-h^{\alpha} \gamma \tau \lambda_{i}\right) \omega$. Applying Lemma 6, we have the following.

(1) By means of $m(0) n^{\prime}(0)-m^{\prime}(0) n(0)=2\left(1+\alpha-h^{\alpha} \gamma \lambda_{i}+\right.$ $\left.2 h^{\alpha} \tau \gamma \lambda_{i}\right)\left(1-h^{\alpha} \gamma \tau \lambda_{i}\right)>0$, we have

$$
\begin{gathered}
1-h^{\alpha} \gamma \tau \lambda_{i}>0, \\
1+\alpha-h^{\alpha} \gamma \lambda_{i}+2 h^{\alpha} \tau \gamma \lambda_{i}>0 .
\end{gathered}
$$


Based on (26), it has

$$
h^{\alpha}<\frac{1}{\gamma \tau \lambda_{i}} .
$$

Discussing (27), if $\tau \geq 0.5$, then (27) comes into existence. If $\tau<0.5$, then it require is

$$
h^{\alpha}<\frac{1+\alpha}{\gamma \lambda_{i}(1-2 \tau)} .
$$

(2) When $2\left(1-h^{\alpha} \gamma \tau \lambda_{i}\right) \neq 0$ from (26), the solution of $n(\omega)=0$ is 0 . Since $\alpha<1$ we have $1-\alpha+h^{\alpha} \gamma \lambda_{i}>0$ and $1+\alpha-h^{\alpha} \gamma \lambda_{i}+2 h^{\alpha} \tau \gamma \lambda_{i}>0$ from (27); then the solutions of $m(\omega)=0$ are $\pm \sqrt{\left(1+\alpha-h^{\alpha} \gamma \lambda_{i}+2 h^{\alpha} \tau \gamma \lambda_{i}\right) /\left(1-\alpha+h^{\alpha} \gamma \lambda_{i}\right)}$. Therefore, the solutions of the related pair $m(\omega)$ and $n(\omega)$ are interlaced.

By comparing the right parts of (28) and (29), we can obtain that when $\tau>1 /(3+\alpha), 1 / \gamma \tau \lambda_{i}<(1+\alpha) / \gamma \lambda_{i}(1-2 \tau)$; otherwise, when $\tau \leq 1 /(3+\alpha), 1 / \gamma \tau \lambda_{i} \geq(1+\alpha) / \gamma \lambda_{i}(1-2 \tau)$. Then, the consensus conditions of the multiagent system are obtained. The proof is finished.

Remark 8. Suppose that the fractional-order $\alpha=1$; we can get the consensus condition $h<1 / \gamma \tau \lambda_{n}$ when $\tau>1 / 4$, and $h<2 / \gamma \lambda_{n}(1-2 \tau)$ when $\tau \leq 1 / 4$, where $\lambda_{n}$ is the maximum eigenvalue of matrix $L$.

Remark 9. Suppose that the communication delay $\tau=0$, we can get $\tau<1 /(3+\alpha)$; therefore, $h<\left((1+\alpha) / \gamma \lambda_{n}\right)^{1 / \alpha}$ where $\lambda_{n}$ is the maximum eigenvalue of matrix $L$. This result is the same as the consensus condition in Corollary 3.

\section{Simulations}

Suppose that the system is composed of four fractional-order dynamical agents (Figure 1). The connection weights between individuals are $a_{21}=a_{12}=0.7, a_{42}=a_{24}=0.8, a_{31}=a_{13}=$ 0.9 , and $a_{14}=a_{41}=1$. Through the network topology of the system, we can get the adjacency matrix

$$
A=\left(\begin{array}{cccc}
0 & 0.7 & 0.9 & 1 \\
0.7 & 0 & 0 & 0.8 \\
0.9 & 0 & 0 & 0 \\
1 & 0.8 & 0 & 0
\end{array}\right)
$$

Suppose that the order of the fractional multiagent dynamics is $\alpha=0.8$ and the system control gain is $\gamma=1$; then we can obtain the relationship between the sampling period and the upper bound of sampling delays (Figure 2) from the conditions in Theorem 7 . In order to make the system meet the condition of reaching consensus, we can set the sampling period according to the sampling delay of the system or decide the stable fields of sampling delays by means of the sampling period. Suppose the order of the fractional multiagent dynamics is $\alpha=0.8$ and the control gain $\gamma=1$; then we can obtain that the upper bound of sampling period $h_{\max }=1.0993 \mathrm{~s}$ corresponding delay $\tau=0.2632 \mathrm{~s}$ from Figure 2.

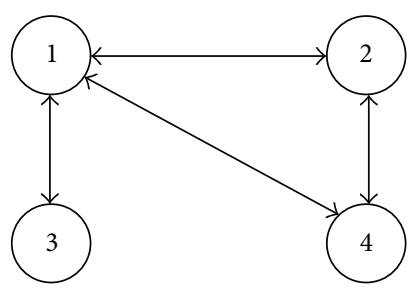

FIGURE 1: Network topology of the multiagent systems.

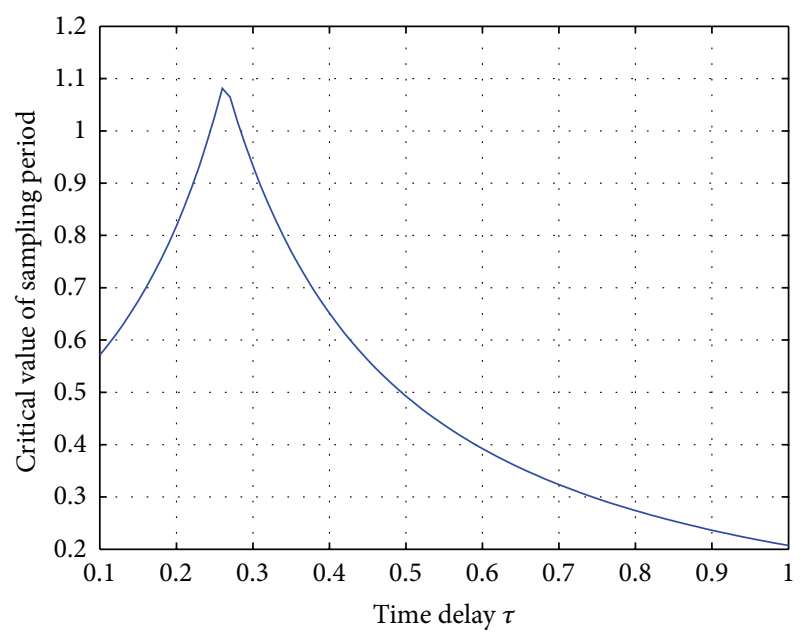

FIGURE 2: Relationship between the communication delay and the upper bound of sampling period.

Simulation 1. Assume the sampling delay of multiagent system is $\tau=0.25 \mathrm{~s}$, we can obtain the upper bound of sampling period is $1.0272 \mathrm{~s}$. In computer simulation, selecting the sampling period $h=0.90 \mathrm{~s}$, the consensus can be asymptotically reached (Figure 3) through fractional-order coordination algorithm.

Simulation 2. Assume the sampled delay of multiagent system is $\tau=0.49 \mathrm{~s}$, the upper bound of sampling period is 0.5054 in Figure 2. In computer simulation, selecting the sampling period $h=0.50 \mathrm{~s}$, the consensus can be asymptotically reached much more slowly with increasing of time delay (Figure 4).

Simulation 3. Assume the sampling delay of multiagent system is $\tau=0.50 \mathrm{~s}$, the upper bound of sampling period is 0.4928 in Figure 2. In computer simulation, selecting the sampling period $h=0.50 \mathrm{~s}$, the movement trajectories of the multiagent systems will be asymptotically diverged and the consensus cannot be reached (Figure 5) through fractionalorder coordination algorithm.

Simulation 4. Let time delay continue increasing, suppose the sampling delay is $\tau=0.80 \mathrm{~s}$, the upper bound of sampling period will be 0.2739 in Figure 2. In computer simulation, selecting the sampling period $h=0.25 \mathrm{~s}$, the movement trajectories of the multiagent systems will be asymptotically converged and the consensus can be reached (Figure 6). 


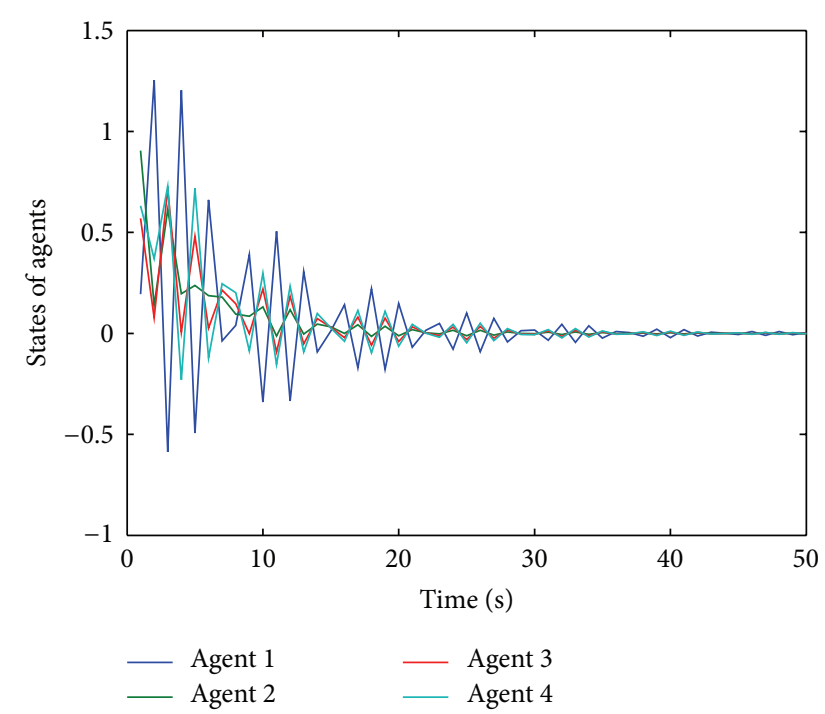

FIGURE 3: Movement trajectories of the multiagent systems with delay $0.25 \mathrm{~s}$ and sampling period $0.90 \mathrm{~s}$.

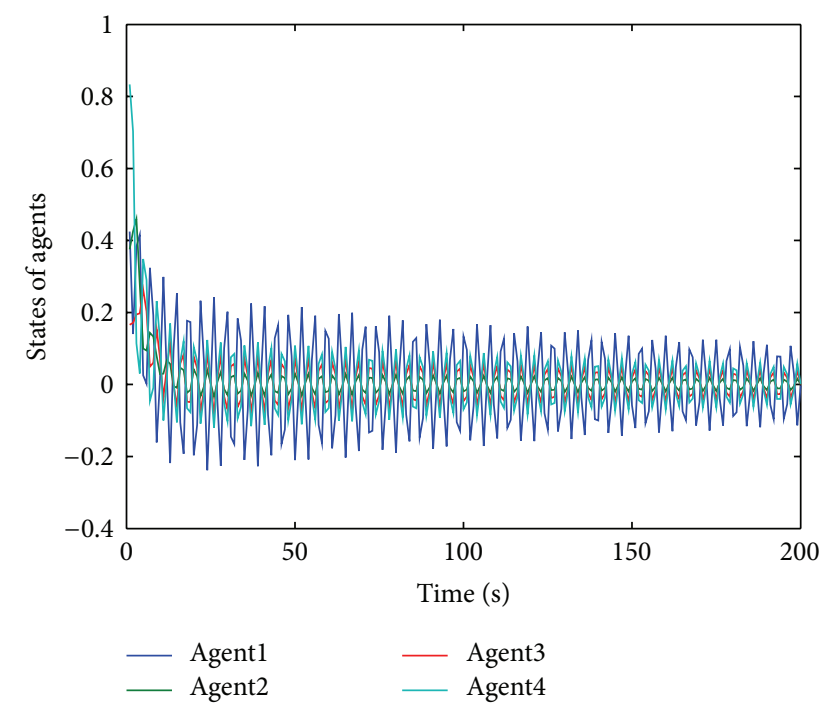

Figure 4: Movement trajectories of the multiagent systems with delay $0.49 \mathrm{~s}$ and sampling period $0.50 \mathrm{~s}$.

Although the sampling period is less than the sampling delay, the consensus can be still achieved under the condition of Theorem 7.

\section{Conclusions}

This paper studies distributed coordination of fractionalorder multiagent system with sampled control and sampling delay. By applying the stability theory of discretetime domain, sampled-data control of FOMAS with directed network topology is investigated, and the upper bound of the sampling period is obtained. Based on the HermiteBiehler Theorem, the collaborative control of fractional-order multiagent systems with sampled data and sampling delay is

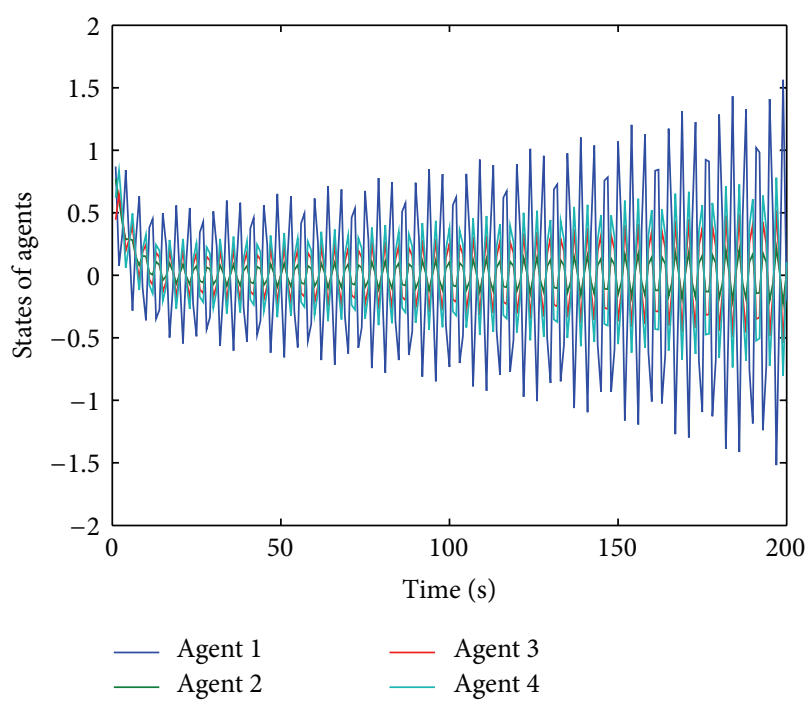

Figure 5: Movement trajectories of the multiagent systems with delay $0.50 \mathrm{~s}$ and sampling period $0.50 \mathrm{~s}$.

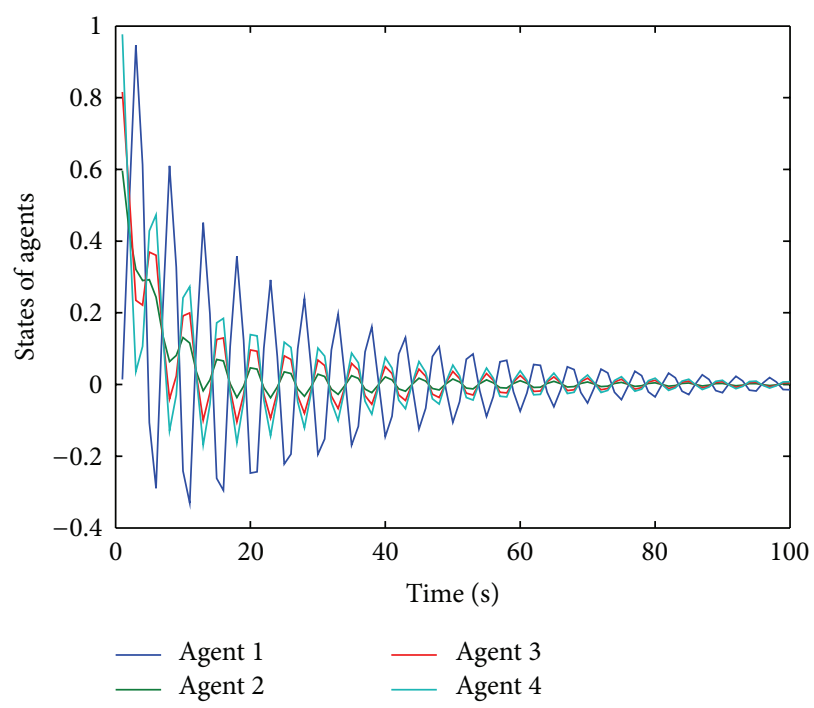

FIGURE 6: Movement trajectories of the multiagent systems with delay $0.80 \mathrm{~s}$ and sampling period $0.25 \mathrm{~s}$.

studied. The relations between sampling delay and sampled period are obtained to ensure the consensus of FOMAS. Research of the robust stability of FOMAS will be carried out in the following work.

\section{Disclosure}

This paper has not been published previously and is not under consideration of publication elsewhere.

\section{Acknowledgments}

This research is supported in part by the State Key Development Program for Basic Research of China (Grant no. 
2012CB720003), the National Natural Science Foundation of China (Grant nos. 61273152, 61127007, 61170161, and 61174085), the Natural Science Foundation of Shandong Province of China (no. ZR2011FM017) and the Science Foundation of China postdoctoral (under Grant no. 2011M500215).

\section{References}

[1] S. Li, H. Du, and X. Lin, "Finite-time consensus algorithm for multi-agent systems with double-integrator dynamics," Automatica, vol. 47, no. 8, pp. 1706-1712, 2011.

[2] P. Lin and Y. Jia, "Consensus of second-order discrete-time multi-agent systems with nonuniform time-delays and dynamically changing topologies," Automatica, vol. 45, no. 9, pp. 21542158, 2009.

[3] J. Yu and L. Wang, "Group consensus in multi-agent systems with switching topologies and communication delays," Systems and Control Letters, vol. 59, no. 6, pp. 340-348, 2010.

[4] F. Chen, Z. Chen, L. Xiang, Z. Liu, and Z. Yuan, "Reaching a consensus via pinning control," Automatica, vol. 45, no. 5, pp. 1215-1220, 2009.

[5] W. Ren, R. W. Beard, and E. M. Atkins, "Information consensus in multivehicle cooperative control," IEEE Control Systems Magazine, vol. 27, no. 2, pp. 71-82, 2007.

[6] H. Yang, Z. Zhang, and S. Zhang, "Consensus of second-order multi-agent systems with exogenous disturbances," International Journal of Robust and Nonlinear Control, vol. 21, no. 9, pp. 945-956, 2011.

[7] D. Meng, Y. Jia, J. Du, and F. Yu, "Tracking control over a finite interval for multi-agent systems with a time-varying reference trajectory," Systems \& Control Letters, vol. 61, no. 7, pp. 807-818, 2012.

[8] Y. Sun and L. Wang, " $H_{\infty}$ consensus of second-order multiagent systems with asymmetric delays," Systems \& Control Letters, vol. 61, no. 8, pp. 857-862, 2012.

[9] G. Xie, H. Liu, L. Wang, and Y. Jia, "Consensus in networked multi-agent systems via sampled control: fixed topology case," in Proceedings of the American Control Conference (ACC '09), pp. 3902-3907, June 2009.

[10] G. Xie, H. Liu, L. Wang, and Y. Jia, "Consensus in networked multi-agent systems via sampled control: switching topology case," in Proceedings of the American Control Conference (ACC '09), pp. 4525-4530, June 2009.

[11] C.-L. Liu and F. Liu, "Stationary consensus of heterogeneous multi-agent systems with bounded communication delays," Automatica, vol. 47, no. 9, pp. 2130-2133, 2011.

[12] Y. Cao, W. Ren, and Y. Li, "Distributed discrete-time coordinated tracking with a time-varying reference state and limited communication," Automatica, vol. 45, no. 5, pp. 1299-1305, 2009.

[13] Y. Gao and L. Wang, "Sampled-data based consensus of continuous-time multi-agent systems with time-varying topology," IEEE Transactions on Automatic Control, vol. 56, no. 5, pp. 1226-1231, 2011.

[14] Z.-J. Tang, T.-Z. Huang, J.-L. Shao, and J.-P. Hu, "Leaderfollowing consensus for multi-agent systems via sampled-data control," IET Control Theory \& Applications, vol. 5, no. 14, pp. 1658-1665, 2011.
[15] W. Yu, W. X. Zheng, G. Chen, W. Ren, and J. Cao, "Second-order consensus in multi-agent dynamical systems with sampled position data," Automatica, vol. 47, no. 7, pp. 1496-1503, 2011.

[16] S. Das, Functional Fractional Calculus for System Identification and Controls, Springer, Berlin, Germany, 2008.

[17] A. A. Kilbas, H. M. Srivastava, and J. J. Trujillo, Theory and Applications of Fractional Differential Equations, vol. 204 of North-Holland Mathematics Studies, Elsevier Science B.V., Amsterdam, The Netherlands, 2006.

[18] J. Sabatier, O. P. Agrawal, and J. A. Tenreiro Machado, Advances in Fractional Calculus: Theoretical Developments and Applications in Physics and Engineering, Springer, 2007.

[19] I. Podlubny, Fractional Differential Equations, vol. 198, Academic Press, San Diego, Calif, USA, 1999.

[20] W. Ren and Y. C. Cao, Distributed Coordination of Multi-Agent Networks, Springer, London, UK, 2011.

[21] Y. Cao, Y. Li, W. Ren, and Y. Chen, "Distributed coordination of networked fractional-order systems," IEEE Transactions on Systems, Man, and Cybernetics, Part B, vol. 40, no. 2, pp. 362370, 2010.

[22] Y. Cao and W. Ren, "Distributed formation control for fractional-order systems: dynamic interaction and absolute/ relative damping," Systems and Control Letters, vol. 59, no. 3-4, pp. 233-240, 2010.

[23] K. Ogata, Discrete-Time Control Systems, Prentice-Hall, Englewood Cliffs, NJ, USA, 1995. 


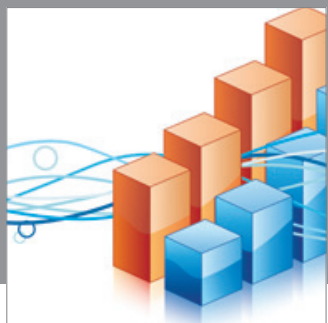

Advances in

Operations Research

mansans

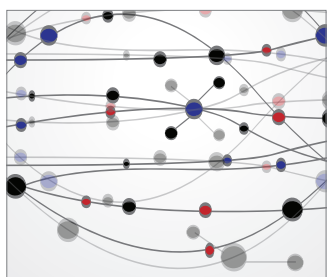

The Scientific World Journal
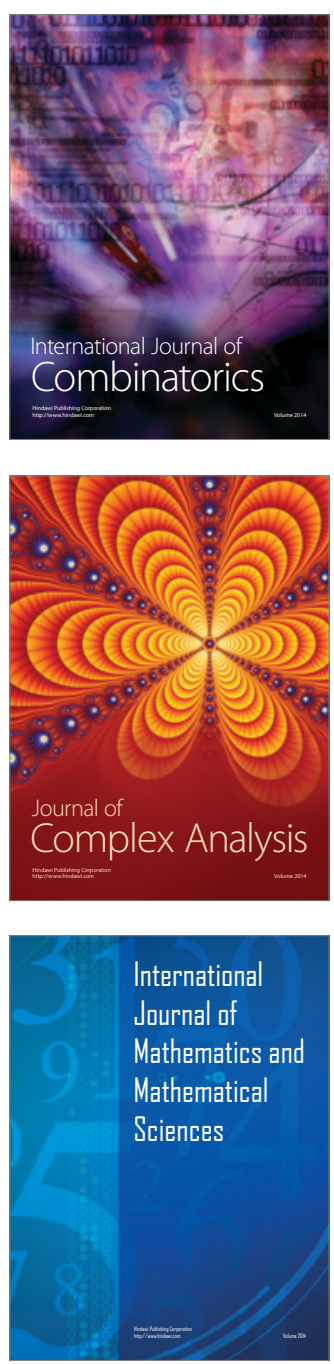
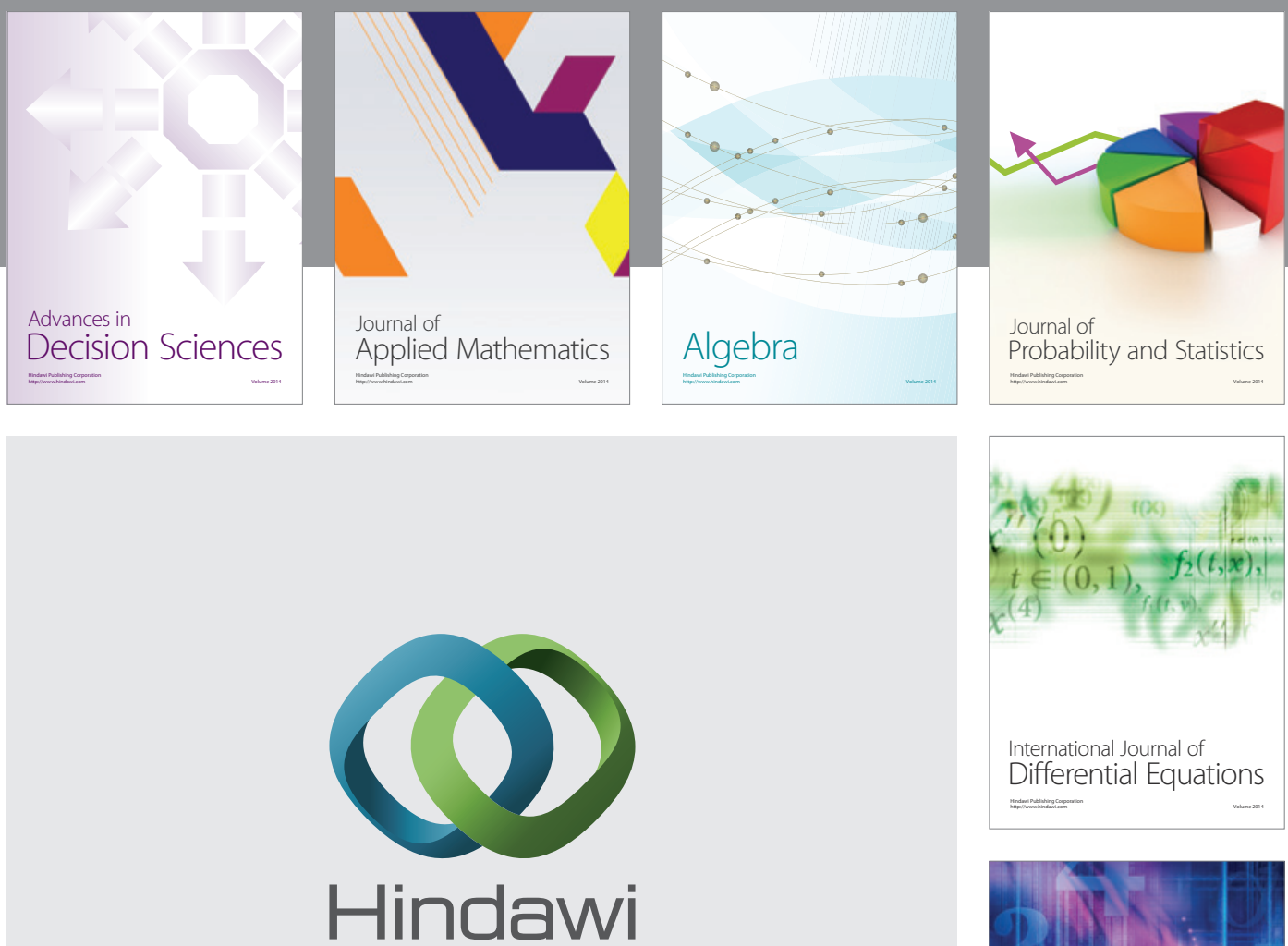

Submit your manuscripts at http://www.hindawi.com
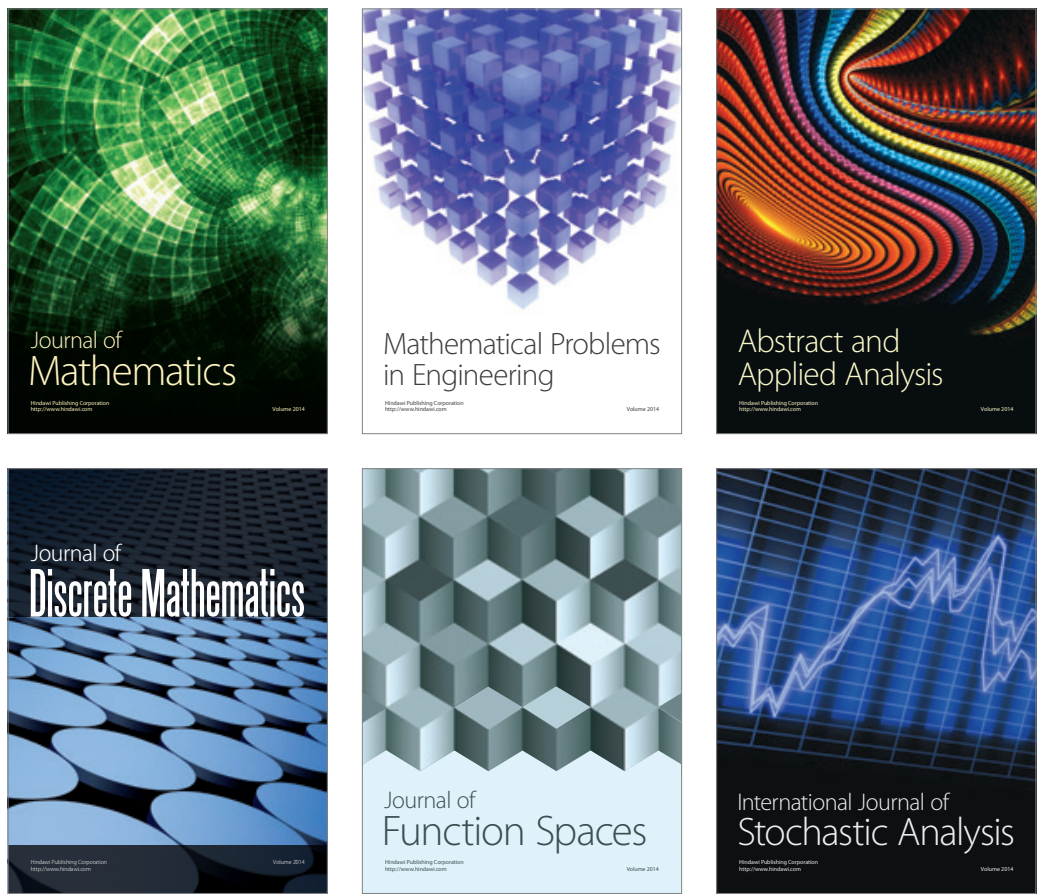

Journal of

Function Spaces

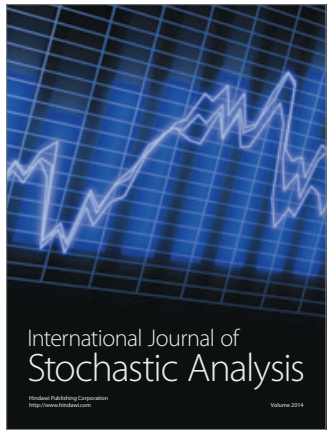

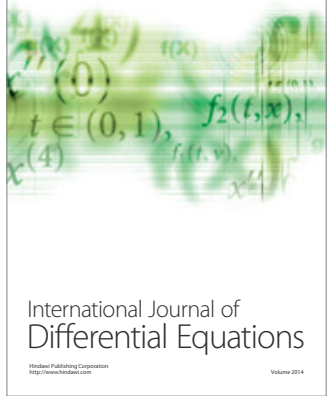
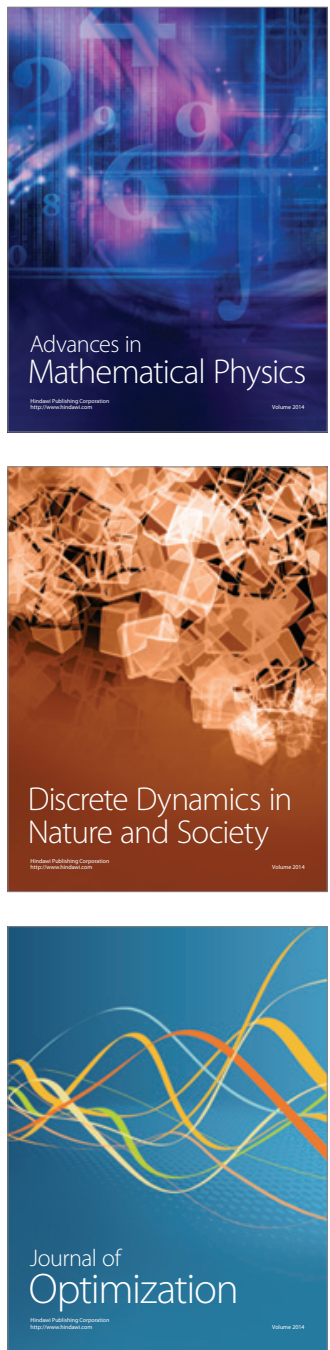